\title{
Leak detection optimisation on retina fluorescein angiography images using phase stretch transform for malaria retinopathy
}

\author{
Febry Putra Rochim*, Hanung Adi Nugroho, Noor Akhmad Setiawan \\ Department of Electrical Engineering and Information Technology, Faculty of Engineering, Universitas Gadjah Mada, \\ Jl. Grafika No. 2 Kampus UGM, Yogyakarta 55281, Indonesia
}

Article history:

Received: 11 May 2018 / Received in revised form: 12 July 2018 / Accepted: 24 September 2018

\begin{abstract}
Malarial Retinopathy (MR) is indicated by retina alteration such as white dots occurrence which is caused by malaria. Leak detection is a key factor of MR's early diagnosis. Inconsistent size and shape of the leakages with the colour contrast that relatively similar with the background. Leak detection's algorithm is one of the most complex algorithms on the fundus image analysis field. Therefore, improving performance in the leakage detection is essential. This study focuses on automated leakage detection on fluorescein angiography (FA) images. The methods used in this study are vessel segmentation, saliency detection, phase stretch transform (PST), optic disk removal and leak detection to extract some features which then classified to correctly validate the leak. From 20 patient data large focal leak images with 31 leak points, 28 of them have been correctly detected. So, the experiment produced the accuracy and specificity of 0.98 and 0.9 , respectively. With the proposed method of this study, there is a potential to enhance the knowledge on MR field in the future.
\end{abstract}

Keywords: malaria disease; retina haemorrhage; phase stretch transform; fluorescein angiography.

\section{Introduction}

Genus Plasmodium is a kind of parasites that cause human malaria. For the specific species, it consists of $P$. ovale, $P$. knowlesi, $P$. falciparum, $P$. malariae and $P$. vivax [1]. Severe anemia of malaria (SA), respiratory disorder (RD), and cerebral malaria (CM) are the common syndromes of malaria. Severe malaria caused by $P$. falciparum can lead to death if it is not correctly handled; since there is a neurological complication as severe as on cerebral malaria. Someone who diagnosed with malaria will more likely have failure medication upon other threatening disease [2]. Parasitemia occurrence on other disease can cause diagnosis of severe malaria become harder.

Malarial retinopathy (MR) related to retinal alteration such as colour changes, retinal bleeding, retinal bleaching and oedema on pupil vessels [3]. Retinal alteration on severe malaria such as bleeding has been observed more than 130 years [4] and the unique signs of retina are described for the first time in the Africa [5]. The probability of cerebral malaria that lead to death can be predicted with the bleeding on the retina [6]. Retinal bleeding usually occurs on the inner of retinal lining; moreover, it also can occur on all retinal linings. The bleeding occurrence on the event location make it looks like noise. Sub-retinal bleeding is observed by the excessive retinal bleeding with secondary retinal ablation [7].

Retinal whitish is a unique sign which can only see on MR

* Corresponding author.

Email: febry.mti15@mail.ugm.ac.id and further occurrence from the white spotting in macula area can help early detection and medication of severe malaria. Macula is an oval-shaped region in the centre of retina and contains $10 \%$ vision. Fovea is found in the centre of macula and contains the highest concentration of conical cells; fovea is a region where 20/20 vision can be acquired. So, the occurrence of white spotting inside the macula region can be more fatal and considered as severe malaria symptoms or other neurological disorder. If the white spotting occurs inside the macula region, it is called macula bleaching. However, when it occurs outside arcade vascular, it is considered as periphery bleaching [8].

Fluorescein angiography (FA) is an imaging kind that used in ophthalmology clinic which provides retinal vascular map structure and function with highlighting the blockage and the leakage of the retinal vessels [9]. MR consists of important signs of Pediatric Cerebral Malaria. MR revealed almost all common different disorder of angiographic vascular for retina condition and because of that, it is easy to use this condition to develop semi-automatic or automatic device to measure retinal disorder quantitatively. In another hand, the similarity between the eye and brain associated malaria brain seems to suggest that the retina may be a good source of potential biomarkers that may cast light on the processes of cerebral malaria disease. Large focal leakage describes one or more loop leakage fields, in which the largest linear diameter is greater than $125 \mu \mathrm{m}$. Large focal leakage is associated with bleeding [10].

According to the previous research $[11,12]$, there is still a gap that can be improved for the accuracy performance by 
using phase stretch transform. With the accuracy obtained is only $92 \%$. A total of 20 FA images which contain 31 leaks are used in this study [12]. Ground truth images are also available. This research focuses on the application of PST method to detect the retinal leakage for diagnosing malaria disease.

\section{Methods}

The proposed automated leakage detection framework consists of four main steps, i.e. vessels segmentation, saliency detection, PST, and leakage detection. The detailed process can be seen in Fig. 1. The description of each step is presented as follows.

\section{(1) Vessel Segmentation}

Some methods used for segmenting vessels are explained as follows:

\section{a) Pre-processing}

Firstly, fluorescein angiography (FA) image is enhanced by using adaptive histogram to obtain brighter vessels than that of on the original image. Then the enhanced image is undergone by median filtering. The filtered image is still containing some noises. To overcome this matter, morphological opening operation is applied, which is involved the ball type of structuring element (SE) with a radius of 35 and $n$ of 15 .

b) Post-processing

Grey threshold binarisation is used to eliminate undesirable area. After that, we apply the technique to drag small objects that are not included in the region of interest (RoI).

\section{(2) Saliency Detection}

By adopting from the previous study, saliency detection is used generate the vessels with region of interest (RoI) [11].

\section{a) Image Color}

Image colour is a technique for displaying data in array $\mathrm{C}$ as an image that uses various colours in colour map. Where, each element of $\mathrm{C}$ determines the colour of 1-pixel image. The resulting image is $m$-by- $n$ grid pixel; where $m$ is the number of columns and $n$ is the number of rows in $\mathrm{C}$. The row and column of index elements determine the appropriate pixel centre.

\section{b) Retinex}

Retinex is a technique to improve the contrast between vessels to the background of the colour map resulted. The results of retinex can be provided differences in colour then processed to get the red channel results. Red channel is the best result for the input process on the next method.

\section{c) Thresholding}

This technique is used to take the highest contrast portion to obtain the result of vessels and RoI as well according to previous retinex contrast. The results of the process can be seen in Fig. 2 (e).

\section{d) Object Detection}

Object detection is the way to get RoI, including of optic disk. It is done by removing the results from Fig. 2 (e) and 2 (b) to generate the Fig. 2 (f). The result of that process leaves RoI with optic disk and small region.

\section{(3) Phase Stretch Transform}

In this session, we use phase stretch transform (PST) that have been applied in the previous study [11]. PST will generate threshold images and followed by a series of morphological operations as post-processing [13]. PST uses a twodimensional phase domain frequency function which is mathematically stated as follows.

$$
A[n, m]=\measuredangle\langle\operatorname{IFFT}\{\bar{K}[p, q] . \bar{L}[p, q] . \operatorname{FFT} 2\{B[n, m]\}\}\rangle
$$

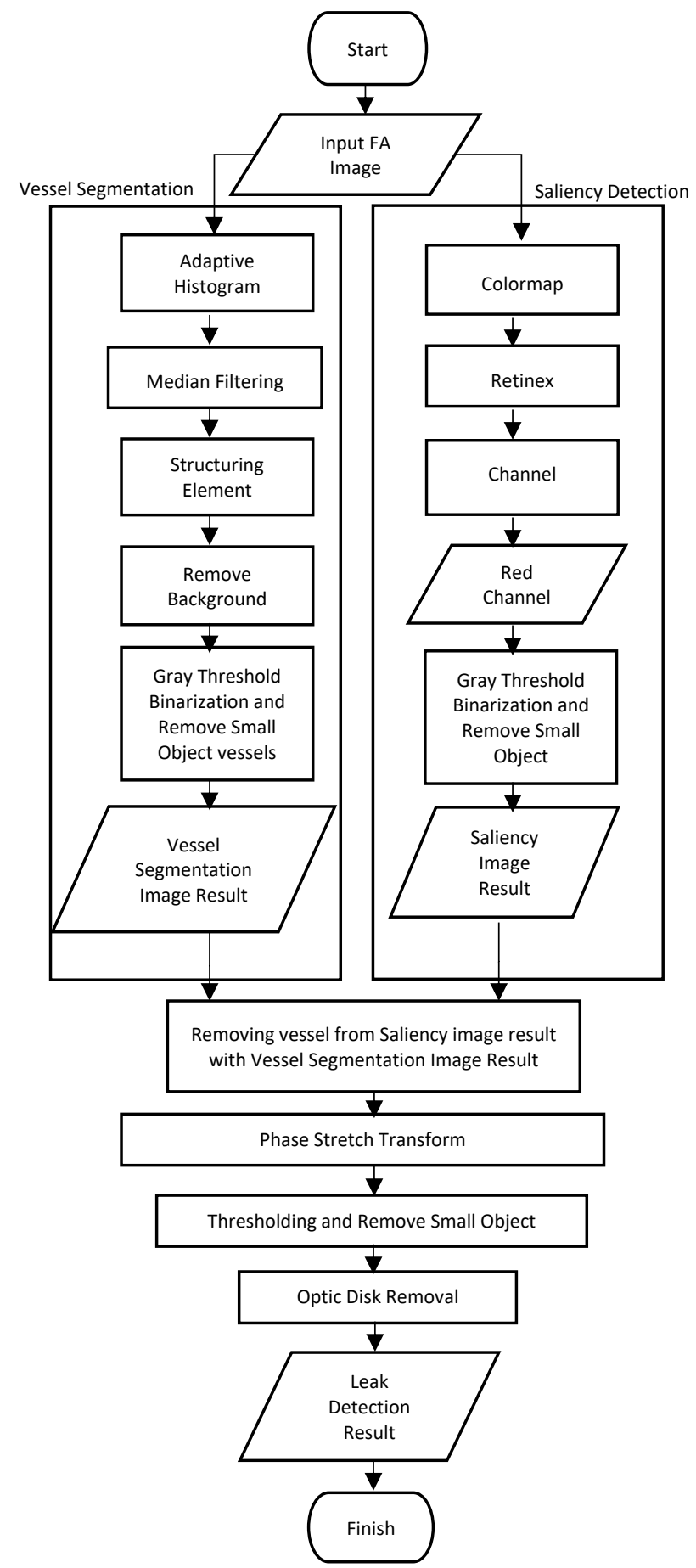

Fig. 1. The flowchart of the proposed approach

The output image is denoted by $A[n, m]$ while the angle of operation is denoted by $\Varangle\langle$.$\rangle . The two-dimensional fast Fourier$ transforms and the inverse two dimensional fast Fourier transforms are expressed as FFT2 and IFFT, respectively. The response of the kernel localization frequency and the non-linear frequency depends on the phases of the kernel curve are 
represented by $\bar{K}[p, q]$ and $\bar{L}[p, q][14]$. The frequency affects the number of stages applied to the image. The lower the frequency component of the image the smaller the phase is applied, and vice versa. PST enhances the edges image by applying larger amount of phase to them, since the edges have higher frequency features.

\section{Results and Discussion}

By using the theory of thresholding output images followed by a series of morphological operation as a post processing can be generate PST image [8]. In this proposed work, the parameter required for low-pass filter and operating morphology is 0.0021 . While the PST and thresholding parameters refer to [8], the power phases of 0.48 and warp power of 12.48 with the minimum and maximum limits are -1 and 0.0019 . The images results of the process are shown in Fig. 2 .

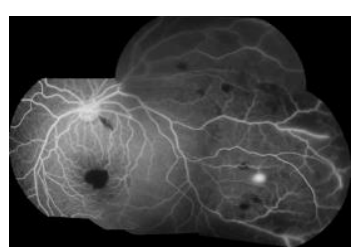

(a)

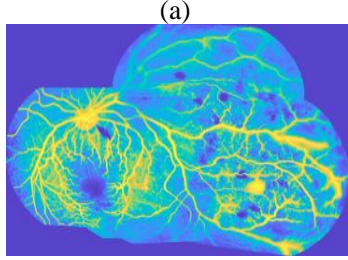

(c)

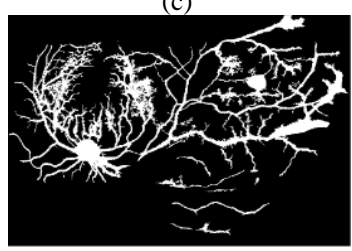

(e)

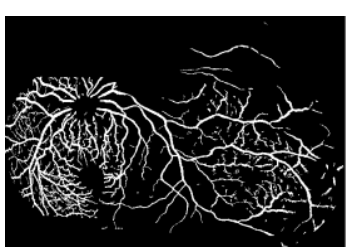

(b)

(d)

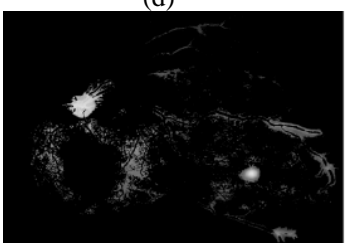

(f)

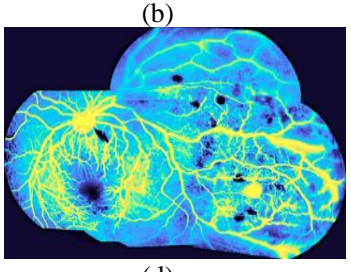

Fig 2. (a) Example FA images. (b) Vessel Segmentation Result. (c) Color Map (d) Retinex of (c). (e) Result of thresholding (d). (f) Removing (e) with (b).

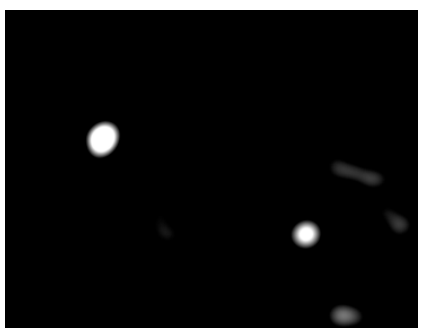

(a)

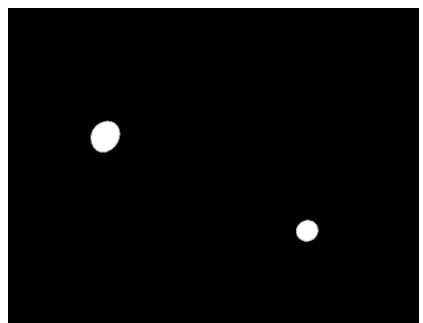

(b)
Fig 3. (a) PST Result. (b) Thresholding Result.

A technique by specifying the object point was used. After some results point from thresholding obtained (as shown in Fig. 3 ), then determine the vessel segmentation result based on the size of object point. Based on the results of the image, each vessel point is then calculated using a histogram. The higher the average histogram value at that point, more vessels have passed through that point. Then, that point can be determined as optic disk. An example result of the OD removal process can be seen in Fig. 4. Finally, performance of the proposed method is evaluated by calculating the accuracy obtained from comparison of detected leak with the ground truth image as shown in Table 1.
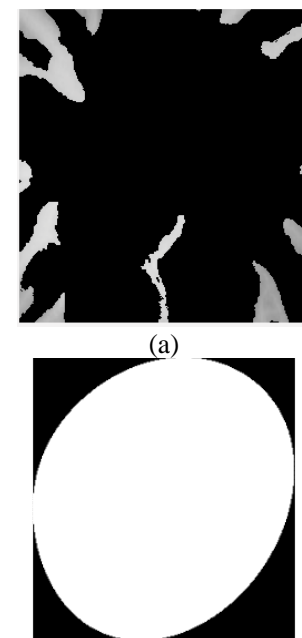

(c)

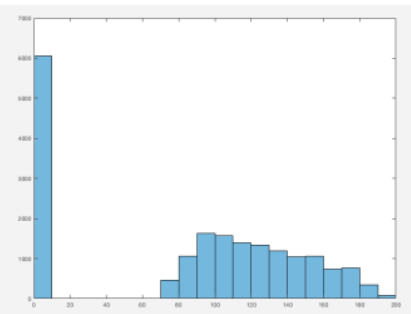

(b)

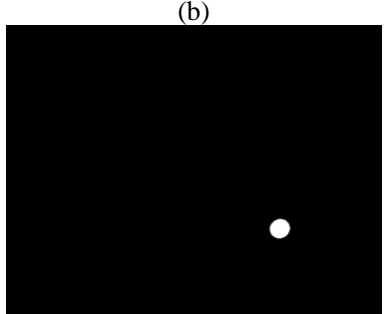

(d)

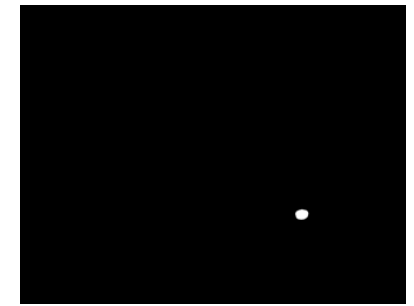

(e)

Fig 4. (a) Vessel of Centroid Object Result. (b) Histogram Result. (c) OD Object Result. (d) Leak Detection Result. (e) Ground Truth

Table 1. Result of performance

\begin{tabular}{cccc}
\hline Images & Accuracy & Ground truth & Detected leak \\
\hline Image 1 & 99 & 1 & 1 \\
Image 2 & 99 & 1 & 1 \\
Image 3 & 99 & 1 & 1 \\
Image 4 & 99 & 1 & 1 \\
Image 5 & 99 & 2 & 1 \\
Image 6 & 96 & 1 & 2 \\
Image 7 & 99 & 2 & 1 \\
Image 8 & 99 & 6 & 2 \\
Image 9 & 97 & 1 & 4 \\
Image 10 & 99 & 3 & 1 \\
Image 11 & 99 & 2 & 2 \\
Image 12 & 99 & 1 & 2 \\
Image 13 & 99 & 1 & 1 \\
Image 14 & 97 & 1 & 1 \\
Image 15 & 99 & 1 & 1 \\
Image 16 & 99 & 1 & 1 \\
Image 17 & 99 & 1 & 1 \\
Image 18 & 99 & 2 & 1 \\
Image 19 & 99 & 1 & 2 \\
Image 20 & 99 & $\mathbf{3 1}$ & $\mathbf{2 8}$ \\
\hline & Total & &
\end{tabular}

\section{Conclusion}

Phase stretch transform (PST) is one of the methods that can be used for leak segmentation in malaria retinopathy (MR). By involving $20 \mathrm{FA}$ images with 31 leak points. This method success determined 28 true points by obtaining the accuracy and specificity of 0.98 and 0.90 , respectively. Since the PST very affected by the pre-processing result, development and 
improvement of pre-processing will conduct in the future to obtain the better final result.

\section{References}

1. World Malarial Report, World Health Organization, 2013

2. H. Reyburn, R. Mbatia, C. Drakeley, I. Carneiro, E. Mwakasungula, Mwerinde, K. Saganda, J. Shao, A. Kitua, R. Olomi, B. M. Green-wood, C. J. Whitt, Overdiagnosis of malaria in patients with severe febrile illness in Tanzania: a prospective study, BMJ 329 (2004) 1212-1217.

3. I. F. Schemann, Doumbo, D. Malvy, L. Traore, A. Kone, T. Sidibe, M. Keita, Ocular lesions associated with malaria in children in Mali, Am. J. Trop. Med. Hyg. 67 (2002) 61-63.

4. F. Poncet, De la retino-choroidite palustre, Ann. Ocul. 79 (1878) 201-218

5. S. Lewallen, T. E. Taylor, M. E. Molyneux, B. A. Wills, Courtright Ocular fundus ndings in Malawian children with cerebral malaria, Ophthalmol. 100 (1993) 857-861.

6. T. Hnscheid, Diagnosis of malaria: a review of alternatives to conventional microscopy, Clin. Lab. Haematol. 21 (1999) 235-245.

7. V. A. White, S. Lewallen, N. A. V. Beare, M. E. Molyneux, T. E. Taylor,
Retinal pathology of pediatric cerebral malaria in Malawi, PloS One 4 (2009) e4317.

8. K. Khurshid et al., Comparison of Niblack inspired Binarization methods for ancient documents, SPIE Conference, vol. 7247, 2009.

9. S. Dithmar and F. Holz. Fluorescence angiography in ophthalmology: Fluorescein angiography, indocyanine green angiography, fundus autofluorescence. Springer-Verlag Berlin Heidelberg, 2008.

10. N. Beare, S. Glover and M. Molyneux, Malarial retinopathy in cerebral malaria, Am. J. Trop. Med. Hyg. 80 (2009) 171.

11. Y. Zhao, I. J. C. MacCormick, D. G. Parry, S. Leach, N. A. V. Beare, S. P. Harding and Y. Zheng, Automated detection of leakage in fluorescein angiography images with application to malarial retinopathy, Sci. Rep. 5 (2015) $1-12$.

12. Y. Zhao, Y. Zheng, Y. Liu, J. Yang, Y. Zhao, D. Chen and Y. Wang, Intensity and Compactness Enabled Saliency Estimation for Leakage Detection in Diabetic and Malarial Retinopathy, IEEE Trans. Med. Imaging 36 (2017) 51-63.

13. K. Firdausy and K. Z. Widhia Oktoeberza, Segmentation of optic disc using dispersive phase stretch transform, 6th Int. Annu. Eng. Semin. Ina., 154-158, 2017.

14. M. H. Asghari and B. Jalali, Physics-inspired image edge detection, in Signal and Information Processing (GlobalSIP), 2014 IEEE Global Conference, 293-296, 2014. 